\title{
Anlotinib inhibits the proliferation, migration and invasion, and induces apoptosis of breast cancer cells by downregulating TFAP2C
}

\author{
FUXIAO FANG ${ }^{1}$ and QING YUAN ${ }^{2}$ \\ ${ }^{1}$ Operating Room, Deqing County People's Hospital, Huzhou, Zhejiang 313200; \\ ${ }^{2}$ Department of Thyroid and Breast Surgery, The Central Hospital of Wuhan, Tongji Medical College, \\ Huazhong University of Science and Technology, Wuhan, Hubei 430014, P.R. China
}

Received April 2, 2021; Accepted July 23, 2021

DOI: $10.3892 / \mathrm{ol} .2021 .13164$

\begin{abstract}
The vascular endothelial growth factor receptor (VEGFR) network contributes to breast cancer pathogenesis and progression. Anlotinib is a highly potent multi-target tyrosine kinase inhibitor that has been previously shown to exert antitumor effects in various types of cancer. The aim of the present study is to investigate the effect of Anlotinib against breast cancer cells in vitro and uncover the possible underlying mechanisms. The human breast cancer cell line MCF-7 was treated with different concentrations of Anlotinib, before cell proliferation, migration, invasion and apoptosis were assessed using colony formation, wound healing, Transwell and TUNEL staining assays. In addition, the expression of transcription factor AP- $2 \gamma$ (TFAP2C) following Anlotinib stimulation was measured using reverse transcription-quantitative PCR and western blot analysis. TFAP2C was overexpressed in MCF-7 using transfection with a pcDNA3.1 vector, before the aforementioned experiments were repeated. The results revealed that Anlotinib impaired cell viability and colony formation, reduced proliferating cell nuclear antigen, Ki-67, MMP2, MMP9 and Bcl-2 expression levels, and inhibited cell migration and invasion. By contrast, the expression levels of tissue inhibitor of metalloproteinase 1, the frequency of apoptotic cells, the expression of Bax and the cleaved caspase-3/caspase- 3 ratio increased in a concentration-dependent manner. Additionally, the expression of TFAP2C decreased after Anlotinib treatment. However, TFAP2C overexpression partially blocked the effects of Anlotinib on the proliferation, migration, invasion and
\end{abstract}

Correspondence to: Dr Qing Yuan, Department of Thyroid and Breast Surgery, The Central Hospital of Wuhan, Tongji Medical College, Huazhong University of Science and Technology, 26 Shengli Road, Jiang'an, Wuhan, Hubei 430014, P.R. China

E-mail: yuanqingyqq@163.com

Key words: Anlotinib, breast cancer, TFAP2C, tyrosine kinase inhibitor, Vandetanib apoptosis of MCF-7 cells. To conclude, Anlotinib suppressed proliferation, migration and invasion, whilst inducing apoptosis of MCF-7 cells, which may be partially dependent on the inhibition of TFAP2C expression.

\section{Introduction}

Malignant tumors of the breast generally occur in the ductal or acinar epithelium. Because of its high morbidity and high mortality, breast cancer is a major disease that seriously impacts on the lives and health of men and women worldwide (1). Surgery is one of the most important treatment options for breast cancer since it is frequently used for breast cancer therapy (2). Although this method can cause deformity or even loss of the breasts of the patient, which can have a great impact on their physical and mental health. Chemotherapy is a reliable method for the treatment of breast malignant tumors. However, multi-drug resistance and metastasis of tumor cells has narrowed the scope of clinical application of chemotherapy drugs to a certain extent (3). Therefore, finding drugs with good curative and anti-metastatic effects in breast cancer has become the focus of breast cancer research.

With the rapid development of life science research, the basic processes of tumor cell signal transduction, cell cycle regulation, apoptosis induction and angiogenesis are gradually being elucidated. Vascular endothelial growth factor receptor (VEGFR) signaling has been identified to play a pivotal role in the regulation of neo-angiogenesis, the process that leads to the formation of new blood vessels from the existing vasculature and that is crucial for tumor growth, invasion and metastasis (4). Therefore, VEGFR has become an important candidate in anti-tumor drug research and a potential target in drug research and development. Drugs directed against the VEGF/VEGFR system can be mainly categorized into two classes, monoclonal antibodies and small-molecule tyrosine kinase inhibitors (TKIs). Since 2018, >20 TKIs have been approved by the Food and Drug Administration (5). These drugs are characterized by high selectivity, high efficacy and low side effects. They are superior to traditional cytotoxicity anti-tumor drugs in the treatment of various cancer types, and some have become first-line drugs for cancer treatment (6). 
Anlotinib is a novel multi-kinase inhibitor that has exhibited efficacy against various types of malignancies in preclinical models, including medullary thyroid cancer, renal cell cancer, gastric cancer and esophageal squamous cell carcinoma (7). It is designed to primarily inhibit VEGFR, platelet-derived growth factor receptors $\alpha$ and $\beta$, fibroblast growth factor receptors, c-Kit and RET (8). Anlotinib is currently undergoing phase-II and/or -III clinical development in China, the USA and Italy for soft tissue sarcomas and various cancer types, such as lung cancer, gastric cancer and thyroid cancer $(9,10)$. Another small-molecule TKI that selectively blocks VEGFR-2, Vandetanib, has been approved for use in 2012 for advanced thyroid cancer and was reported to inhibit cell growth and angiogenesis in breast cancer cells (11). Moreover, the effect of Vandetanib on the proliferation and tumor growth response of breast cancer cells was dependent on transcription factor AP- $2 \gamma$ (TFAP2C) expression (12). A recent study has demonstrated that Anlotinib has good efficacy and low toxicity in a phase-II study evaluating Anlotinib in pre-treated HER-2 negative metastatic breast cancer (13). Additionally, Zhang et al (14) suggested that Anlotinib combined with neoadjuvant chemotherapy had good efficacy and safety in the treatment of occult breast cancer and may represent a potential therapeutic option. TFAP2C is a member of the AP-2 transcription factor family, which has been shown to play a critical role in multiple subtypes of breast cancer (15). In breast cancer, data mining of microarray expression studies has shown an association between high levels of TFAP2C mRNA and higher clinical grade and shorter survival times $(16,17)$. However, the effects of Anlotinib on TFAP2C on the progression of breast cancer remain to be elucidated. The aim of the present study was to explore the antitumor effect and mechanism of action of Anlotinib in the human breast cancer cell line MCF-7. Whether Anlotinib could inhibit MCF-7 cell proliferation and migration by regulating TFAP2C expression was also explored.

\section{Materials and methods}

Cell culture and treatment. The human breast cancer cell line MCF-7 was purchased from the American Type Culture Collection and cultured in RPMI-1640 medium supplemented with $10 \% \mathrm{FBS}$ and $1 \%$ penicillin and streptomycin at $37^{\circ} \mathrm{C}$ with $5 \% \mathrm{CO}_{2}$ (all from Gibco; Thermo Fisher Scientific, Inc.).

Cell transfection. The full length of the TFAP2C sequence was cloned into the pcDNA3.1 vector by Gene Script Biotech Co., Ltd. to construct $2 \mu \mathrm{g}$ TFAP2C overexpression vector (Ov-TFAP2C). The empty vector pcDNA3.1 $(2 \mu \mathrm{g})$ was used as a negative control (Ov-NC). Cells were cultured overnight to reach $60-70 \%$ confluence before transfection. The plasmid transfection was performed using Lipofectamine ${ }^{\circledR} 2000$ (Invitrogen; Thermo Fisher Scientific, Inc.) according to the manufacturer's instructions. Cells were incubated with $5 \% \mathrm{CO}_{2}$ at $37^{\circ} \mathrm{C}$ and were used in subsequent experiments $48 \mathrm{~h}$ post-transfection.

Cell viability measurement. Cell viability was measured using Cell Counting Kit-8 (CCK-8; Beyotime Institute of Biotechnology) assays. Control or transfected MCF-7 cells were seeded in 96-well plates at $5 \times 10^{3}$ cells/well and incubated at $37^{\circ} \mathrm{C}$ for $24 \mathrm{~h}$. The cells were the treated with different concentrations of Anlotinib (0, 2, 4 and $6 \mu \mathrm{M}$; >99\%; Selleck Chemicals) at $37^{\circ} \mathrm{C}$ for $24 \mathrm{~h}$. A total of $10 \mu \mathrm{l} \mathrm{CCK}-8$ solution was added to each well for $2 \mathrm{~h}$ of culture at $37^{\circ} \mathrm{C}$. Cell viability was detected using a microplate reader (Bio-Rad Laboratories, Inc.) at the absorbance of $450 \mathrm{~nm}$.

Colony formation assay. For colony formation assays, control or transfected MCF-7 cells ( $1 \times 10^{3}$ cells) were resuspended in $1 \mathrm{ml}$ medium, then cultured in 24 -well plates overnight. The attached cells were treated with Anlotinib $(0,2,4$ and $6 \mu \mathrm{M})$ at $37^{\circ} \mathrm{C}$ for $24 \mathrm{~h}$, then cultured in complete RPMI-1640 medium for an additional 2 weeks. After fixation with $20 \%$ methanol for $30 \mathrm{~min}$ at room temperature, $0.5 \%$ crystal violet was used to stain cells for $5 \mathrm{~min}$ at room temperature and the number of colonies with $>50$ cells was counted manually using a light microscope (magnification, x100).

Wound healing assay. Control or transfected MCF-7 cells were seeded onto 6 -well plates $\left(4 \times 10^{5}\right.$ cells/well) and cultured in normal conditions to $70-80 \%$ confluence. The cell surface was scratched with a $100-\mu 1$ pipette tip to create an artificial wound, and the medium was replaced with serum-free RPMI-1640 containing Anlotinib (0, 2, 4 and $6 \mu \mathrm{M})$ and culturing for $24 \mathrm{~h}$ at $37^{\circ} \mathrm{C}$. The cell migration was observed with images captured in each group at 0 and $24 \mathrm{~h}$ using a light microscope (magnification, x100; Olympus Corporation). The cell migration rate was calculated using the following equation: (Initial width at $0 \mathrm{~h}$ - final width at $24 \mathrm{~h}$ )/Initial width at $0 \mathrm{~h}$. The relative migration rate was obtained by normalizing to the control group.

Transwell assay. For Transwell analysis, an 8- $\mu \mathrm{m}$ Transwell chamber (Corning, Inc.) was coated with $250 \mathrm{mg} / \mathrm{ml} \mathrm{\text {Matrigel } ^ { \mathrm { TM } }}$ (BD Biosciences) at room temperature for $24 \mathrm{~h}(18-20)$, then placed into a 24-well plate. The upper chambers were seeded with control or transfected MCF-7 cells suspended in $200 \mu 1$ serum-free DMEM at a density of $1 \times 10^{5}$ following Anlotinib treatment. RPMI-1640 medium containing 10\% FBS was added to the lower chamber for $24 \mathrm{~h}$ of incubation at $37^{\circ} \mathrm{C}$. Finally, the invading cells into the bottom of chamber were fixed with $4 \%$ methanol at $37^{\circ} \mathrm{C}$ for $10 \mathrm{~min}$, then stained with $0.1 \%$ crystal violet solution at $37^{\circ} \mathrm{C}$ for $15 \mathrm{~min}$. The number of invasive cells in five random fields were counted using a light microscope (magnification, x100).

TUNEL staining. Cell apoptosis was detected using the TUNEL assay kit (Abcam) following the manufacturer's instructions. The control or transfected MCF-7 cells were fixed with $1 \%$ paraformaldehyde at room temperature for $15 \mathrm{~min}$ after Anlotinib treatment, and then treated with $70 \%$ ethanol on ice. After being exposed to the TUNEL detection reagent for $1 \mathrm{~h}$ at $37^{\circ} \mathrm{C}$, cells were treated with $10 \mu \mathrm{g} / \mathrm{ml}$ DAPI (Shanghai Haoyang Bio Technology Co., Ltd.) to stain the nucleus at $37^{\circ} \mathrm{C}$ for 2-3 min and mounted in an anti-fade reagent (Beijing Solarbio Science \& Technology Co., Ltd.). In total, three fields of view were selected at random, each with $\sim 300-500$ cells and the images were captured using a fluorescence microscope (magnification, x100; Olympus Corporation) and analyzed using the ImageJ software (version $1.48 \mathrm{v}$; National Institutes of Health). 
Western blot analysis. Cells were lysed with RIPA buffer (Beyotime Institute of Biotechnology) containing protease inhibitors (Roche Diagnostics), and protein concentration was quantified using a bicinchoninic-acid assay kit (Thermo Fisher Scientific, Inc.). Equal amount of protein samples $(20 \mu \mathrm{g})$ were separated $12 \%$ SDS-PAGE on $12 \%$ gels, then transferred onto PVDF membranes (EMD Millipore). After being blocked with 5\% non-fat milk for $2 \mathrm{~h}$ at room temperature (diluted with PBS-0.1\% Tween-20), membranes were incubated overnight at $4{ }^{\circ} \mathrm{C}$ with the primary antibodies against proliferating cell nuclear antigen (PCNA; 1:5,000, cat. no. ab29; Abcam), Ki-67 (1:1,000, cat. no. ab92742; Abcam), matrix metalloproteinase (MMP)2 (1:1,000, cat. no. ab92536; Abcam), MMP9 (1:1,000, cat. no. ab76003; Abcam), tissue inhibitor of metalloproteinase-1 (TIMP-1; 1:1,000, cat. no. ab211926; Abcam), Bcl-2 (1:2,000, cat. no. ab32124; Abcam), Bax (1:5,000, cat. no. ab32503; Abcam), cleaved caspase-3 (1:500 cat. no. ab32042; Abcam), caspase-3 (1:5,000, cat. no. ab32351; Abcam), TFAP2C (1:1,000, cat. no. ab218107; Abcam), GAPDH (1:5,000, cat. no. ab8245; Abcam) and the corresponding secondary HRP-conjugated goat anti-rabbit IgG (1:5,000, cat. no. 7074; Cell signaling technology) at room temperature for $2 \mathrm{~h}$, followed by visualization with the Pierce ${ }^{\mathrm{TM}}$ enhanced chemiluminescence western blotting substrate (Thermo Fisher Scientific, Inc.) and quantitative analysis with the ImageJ software (version $1.48 \mathrm{v}$; National Institutes of Health).

Reverse transcription-quantitative PCR (RT-qPCR). Total RNA was extracted from cells using TRIzol ${ }^{\circledR}$ (Invitrogen; Thermo Fisher Scientific, Inc.) according to the manufacturers protocol. A total of $5 \mu \mathrm{g}$ RNA was then reverse transcribed into cDNA using PrimerScript ${ }^{\mathrm{TM}}$ RT Master Mix (cat. no. RR036A; Takara Bio, Inc.). The temperature protocol was $37^{\circ} \mathrm{C}$ for $15 \mathrm{~min}$ and reaction at $85^{\circ} \mathrm{C}$ for $5 \mathrm{sec}$. The qPCR reactions were performed using TB Green ${ }^{\circledR}$ Premix Ex Taq ${ }^{\mathrm{TM}}$ (cat.no. RR420A; Takara Bio, Inc.) on an ABI Prism 7500 sequence detector (Applied Biosystems, Inc.; Thermo Fisher Scientific, Inc.). The thermocycling conditions: Pre-denaturation at $95^{\circ} \mathrm{C}$ for $30 \mathrm{sec}$, followed by denaturation at $95^{\circ} \mathrm{C}$ for $10 \mathrm{sec}$ and annealing at $55^{\circ} \mathrm{C}$ for $30 \mathrm{sec}$ for 40 cycles. The specific primers used were as follows: TFAP2C forward, 5'-ATCGAAAAATGGAGGCC GGT-3' and reverse, 5'-CGGCTTCACAGACATAGGCA-3'; PCNA forward, 5'-GTTACTGAGGGCGAGAAGCG-3' and reverse, 5'-AAGTCTAGCTGGTTTCGGCT-3'; Ki-67 forward, 5'-CTGACCCTGATGAGAGTGAGGGA-3' and reverse, 5'-GGACAGGTGGAGTGTGCATTA-3' and GAPDH forward, 5'-GCAACCGGGAAGGAAATGAATG-3' and reverse, 5'-CCCAATACGACCAAATCAGAGA-3'. Results were normalized to GAPDH expression, and the comparative $\mathrm{Cq}$ method $\left(2^{-\Delta \Delta \mathrm{Cq})}\right.$ was used to calculate the relative gene expression levels (21).

Statistical analysis. GraphPad Prism 8 (GraphPad Software, Inc.) was used for data analysis. Data were generated from three independent experimental repeats. The results are presented as the mean \pm standard deviation. Data analysis was carried out using one-way analysis of variance followed by Tukey's post hoc test. $\mathrm{P}<0.05$ was considered to indicate a statistically significant difference.

\section{Results}

Anlotinib inhibits the proliferation, migration and invasion of breast cancer cell line MCF-7 in a concentration-dependent manner. Different concentrations of Anlotinib (0, 2, 4 and $6 \mu \mathrm{M})$ were used to treat MCF-7 cells, and cell viability, colony formation and PCNA and $\mathrm{Ki}-67$ protein expression were assessed. As shown in Fig. 1A, cell viability was significantly reduced following 2,4 and $6 \mu \mathrm{M}$ Anlotinib treatment. Moreover, the protein expression of PCNA and Ki-67 was significantly reduced by Anlotinib (Fig. 1B). Anlotinib also significantly reduced the number of colonies in MCF-7 cells (Fig. 1C). Furthermore, wound healing and Transwell assays were used to evaluate the effect of Anlotinib on cell migration and invasion, respectively. As demonstrated in Fig. 1D and E, both the cell migration and invasion rate were significantly inhibited by Anlotinib. Moreover, Anlotinib resulted in a significant decrease in MMP2 and MMP9 expression levels and a significant increase in TIMP-1 expression, further indicating an inhibitory effect on cell migration (Fig. 1F). Thus, Anlotinib exerted a concentration-dependent inhibitory effect on MCF-7 cell proliferation, migration and invasion.

Anlotinib promotes the apoptosis of MCF-7 breast cancer cells. Subsequently, the effect of Anlotinib on MCF-7 cells apoptosis was evaluated using TUNEL staining and western blot assays. As shown in Fig. 2A, cells exposed to 2, 4 and $6 \mu \mathrm{M}$ Anlotinib exhibited a higher frequency of TUNEL-positive (apoptotic) cells compared with control $(0 \mu \mathrm{M})$ cells, indicating that Anlotinib could promote apoptosis. This was further evidenced by the decrease in Bcl-2 expression, as well as the increase in Bax expression and cleaved-caspase-3/caspase-3 ratio following treatment with 2, 4 and $6 \mu \mathrm{M}$ Anlotinib (Fig. 2B).

TFAP2C overexpression partially blocks the effect of Anlotinib on MCF-7 cell proliferation, migration and apoptosis. Next, the effect of Anlotinib on TFAP2C expression was determined. As shown in Fig. 3A and B, both the mRNA and protein expression levels of TFAPC2 were reduced in response to Anlotinib treatment, and the effect was concentration-dependent.

TFAP2C was overexpressed in MCF-7 cells (Fig. 3C and D) to explore the changes in the effect of Anlotinib on MCF-7 cells proliferation, migration and apoptosis. Cell viability was higher in Anlotinib + Ov-TFAPC2 group compared with that in the Anlotinib + Ov-NC group (Fig. 3E). In addition, Anlotinib inhibited the expression of PCNA and Ki-67, whereas TFAP2C overexpression partially rescued PCNA and Ki-67 expression (Fig. 3F and G). Similarly, the decreased number of colony-forming units caused by Anlotinib was also significantly increased by TFAP2C overexpression (Fig. 3H). These data revealed the protective effects of TFAP2C overexpression against Anlotinib-induced reduction in $\mathrm{MCF}-7$ cell viability. Finally, TFAP2C-overexpressing MCF-7 cells were exposed to $4 \mu \mathrm{M}$ Anlotinib, then cell migration, invasion and apoptosis were assessed. As shown in Fig. 4A and B, the reduced ability of migration and invasion caused by Anlotinib was reversed following TFAP2C overexpression. Consistently, TFAP2C overexpression also blocked the effect of Anlotinib on MMP2, MMP9 and TIMP-1 expression (Fig. 4C). Furthermore, TFAP2C overexpression significantly reversed 

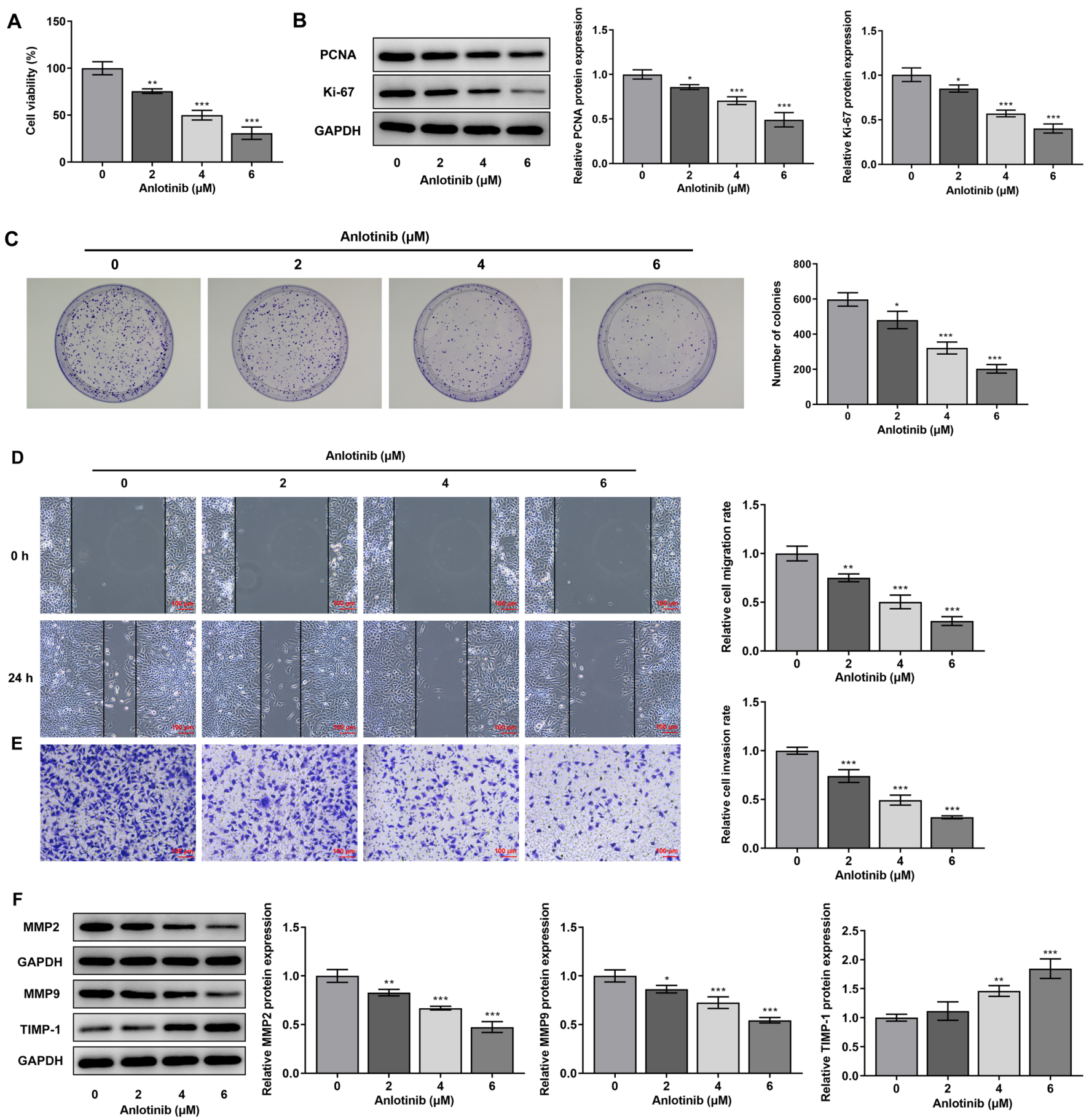

Figure 1. Anlotinib inhibits the proliferation and migration of MCF-7 cells. MCF-7 cells were cultured in medium containing $0,2,4$ or $6 \mu \mathrm{M}$ Anlotinib for 24 h. (A) Cell viability was tested using a Cell Counting Kit-8 assay. (B) PCNA and Ki-67 protein expression was measured by western blotting. (C) Cell proliferation was evaluated using colony formation assays. (D) Cell migration was determined using wound healing and Transwell assays. Scale bars, $100 \mu \mathrm{m}$. (E) which were quantified. (F) MMP2, MMP9 and TIMP-1 protein expression was detected by western blotting. ${ }^{*} \mathrm{P}<0.05,{ }^{* *} \mathrm{P}<0.01$ and ${ }^{* * *} \mathrm{P}<0.001$ vs. $0 \mu \mathrm{M}$ Anlotinib. PCNA, proliferating cell nuclear antigen; TIMP-1, tissue inhibitor of metalloproteinase 1.

the effect of Anlotinib on the frequency of apoptotic cells, as well as on the protein expression of $\mathrm{Bcl}-2$, Bax and the cleaved-caspase-3/caspase-3 ratio (Fig. 5A and B).

\section{Discussion}

In 2018, the latest data released by the International Agency for Research on Cancer showed that among all cancer types, the incidence and mortality of breast cancer ranked second (22).
Breast cancer is also one of the most important causes of death in women over 45 years of age with malignant tumors (23). In most breast cancer cases, patients have excellent prognosis, whereas $\sim 40 \%$ of breast cancer patients undergoing treatment experience recurrence and metastasis (24). The 5-year survival rate of patients with metastatic breast cancer is not higher than $60 \%$, and most patients die due to locoregional recurrence and distant metastasis (25). Therefore, the development of novel effective therapeutic agents for breast cancer remains an 
A
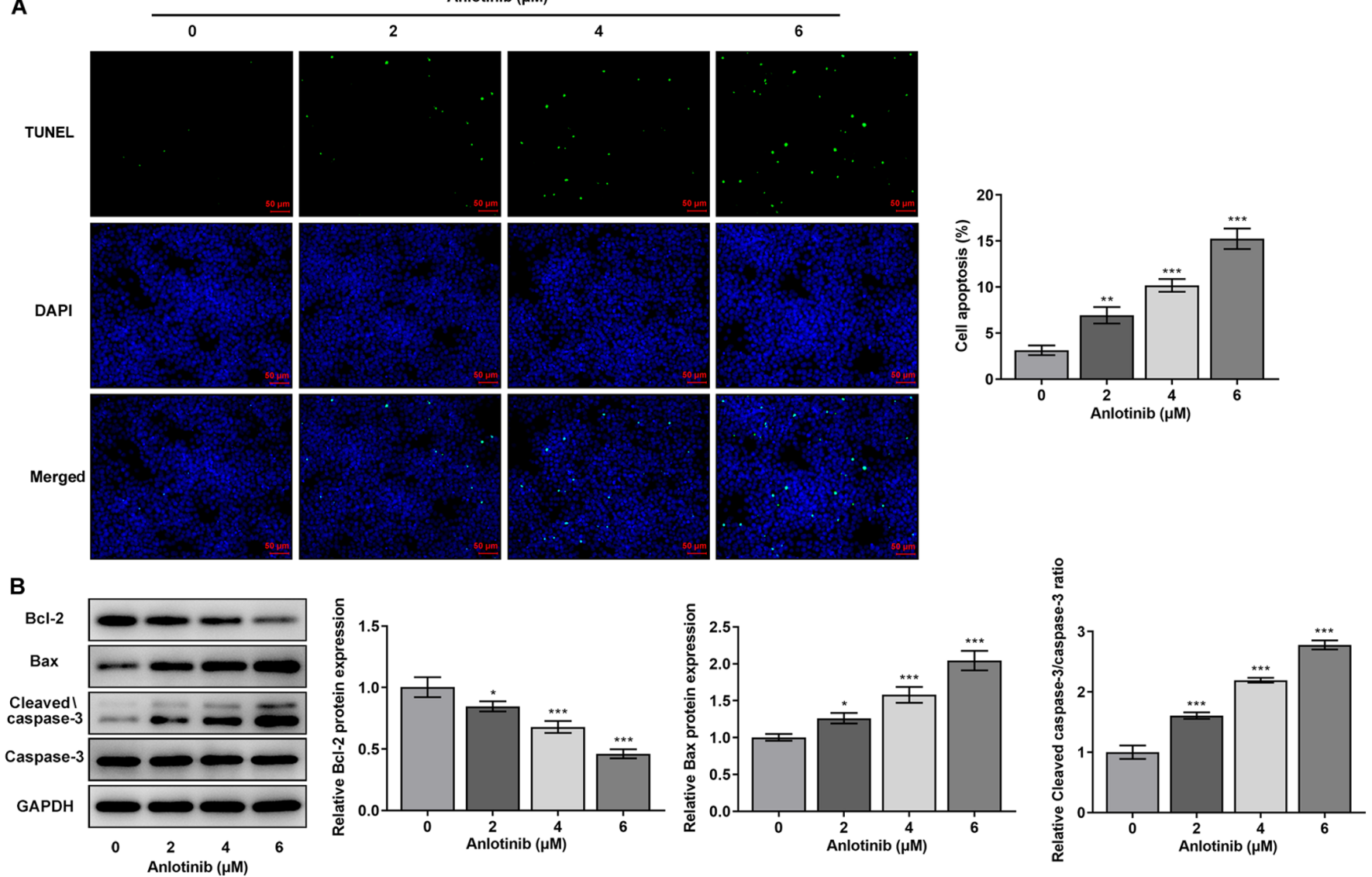

Figure 2. Anlotinib induces the apoptosis of MCF-7 cells. MCF-7 cells were cultured in medium containing 0, 2, 4 or $6 \mu$ M Anlotinib for 24 h. (A) Apoptosis was assessed using TUNEL staining. Scale bar, $50 \mu \mathrm{m}$. (B) Bcl-2, Bax, cleaved caspase-3 and caspase-3 protein expression was measured by western blotting. Data were obtained from three independent experiments. ${ }^{*} \mathrm{P}<0.05,{ }^{* *} \mathrm{P}<0.01$ and ${ }^{* * *} \mathrm{P}<0.001$ vs. $0 \mu \mathrm{M}$ Anlotinib.

urgent medical requirement. In the present study, the potential therapeutic effect of the novel multi-kinase inhibitor Anlotinib was evaluated in breast cancer cells. The results suggested that Anlotinib had an antitumor effect on breast cancer cells by inhibiting their proliferation, migration, invasion and inducing their apoptosis in vitro.

TFAP2C regulates the expression of estrogen receptor $\alpha$ $(\mathrm{ER} \alpha)$, which is transcriptionally regulated in breast cancer (26). Thus, the involvement of TFAP2C in breast cancer has been extensively studied $(12,26,27)$. For example, higher TFAP2C expression has been shown to correlate with poor overall survival after 10 years of diagnosis in $\mathrm{ER} \alpha^{+}$and endocrine therapy-treated subgroups of breast cancer (28). TFAP2C also predicts the outcome in HER2 ${ }^{+}$breast cancer (29). In the present study, Anlotinib inhibited the expression of TFAP2C in a concentration-dependent manner. In luminal breast cancer, the response of cancer cells to Vandetanib is mediated by the TFAP2C target genes epidermal growth factor receptor (EGFR) and RET (12). Similarly, Anlotinib can inhibit the expression of RET, which is a downstream target of TFAP2C in breast cancer (30). Thus, it was hypothesized that Anlotinib may exert its antitumor effect on breast cancer cells by downregulating TFAP2C expression. TFAP2C was overexpressed in MCF-7 cells, which were then subjected cells to Anlotinib treatment. In accordance with our hypothesis, the data revealed that TFAP2C overexpression markedly blocked the antitumor effect of Anlotinib on $\mathrm{MCF}-7$ breast cancer cells. However, the specific signaling pathways downstream of Anlotinib/TFAP2C have not been investigated in this study. TFAP2C is the key regulator of hormone responsiveness in breast cancer cells through the control of multiple pathways of estrogen signaling $(25,30,31)$. Whether these pathways are involved in the effect of Anlotinib on breast cancer via TFAP2C remains to be elucidated. In addition, the conclusion of this study only came from in vitro experiments, lacking the validation of in vivo animal models. The effects of knockdown by using small interfering RNA targeting TFAP2C or overexpression of TFAP2C in MCF-7 cells (without Anlotinib treatment) on cell growth, cell migration, invasion, and cell apoptosis will be investigated in future experiments. Moreover, the use of only a TUNEL assay to detect apoptotic cells is another limitation of this study. Future research should aim to strengthen the conclusions of this study using comprehensive analysis and uncover the underlying mechanisms. For instance, whether signaling pathways is associated with Anlotinib in breast cancer should be analyzed.

In conclusion the present study demonstrated that Anlotinib exerts antitumor effects on breast cancer cells through inhibition of cell growth, induction of apoptosis and suppression of cell migration and invasion. Importantly, the effect Anlotinib against breast cancer might be partially dependent on inhibiting TFAP2C expression. Thus, the finding that Anlotinib may be used as an effective antitumor agent for breast cancer treatment was further confirmed. 
A

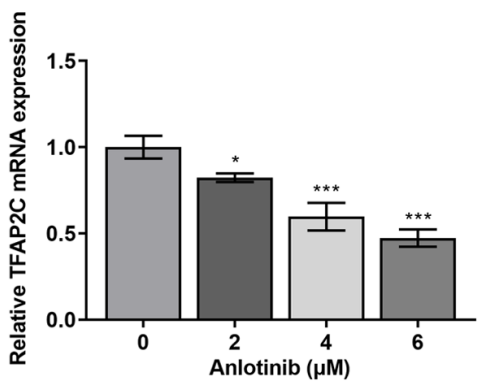

C

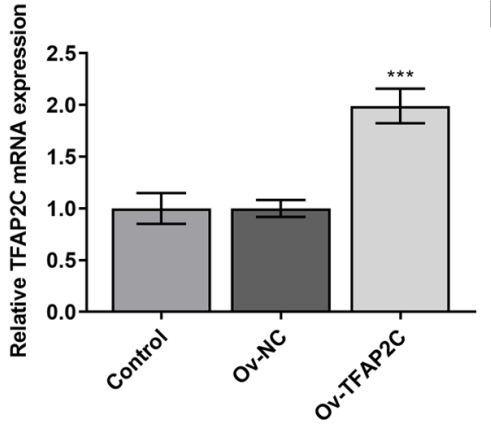

E

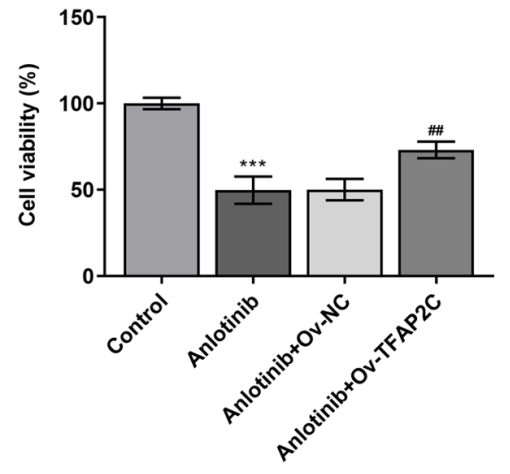

B

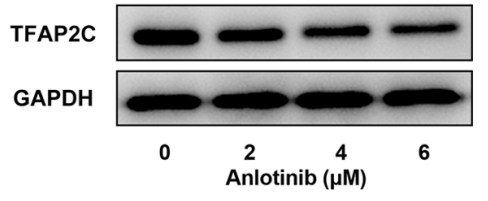

D

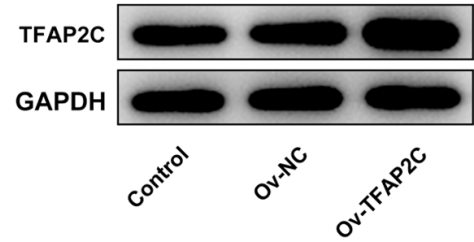

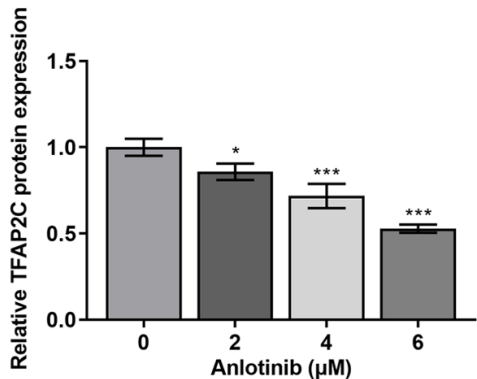

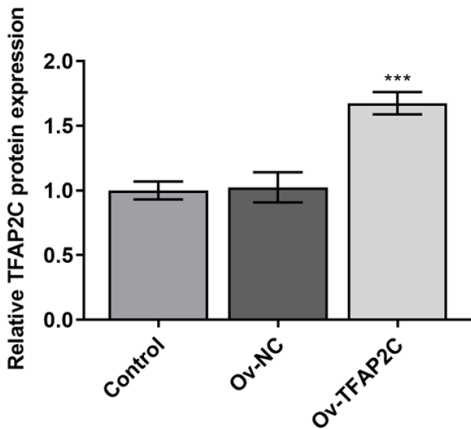

$\mathbf{F}$

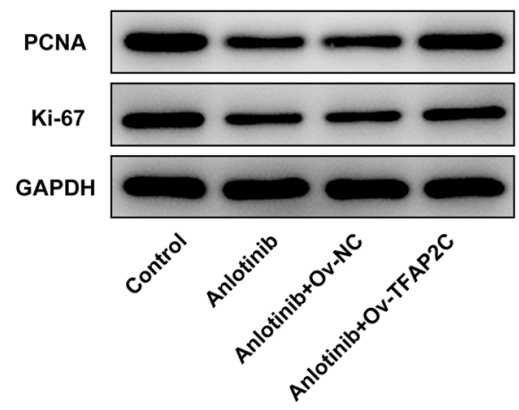

G
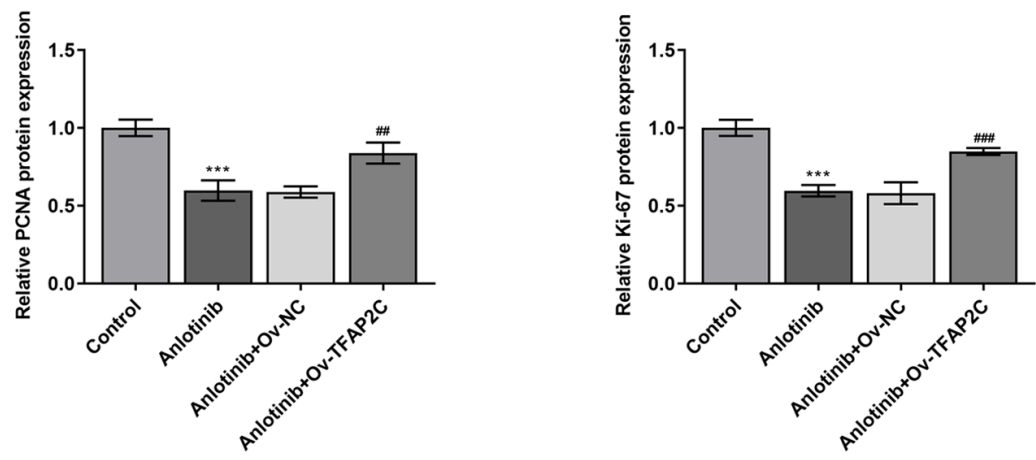

H
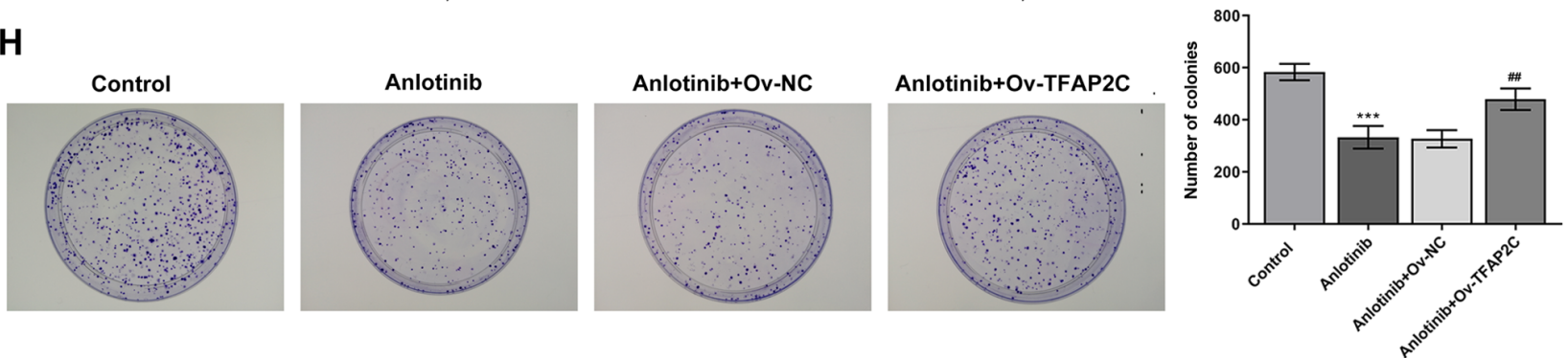

Figure 3. TFAP2C overexpression blocks the inhibitory effect of Anlotinib on MCF-7 cell proliferation. MCF-7 cells were cultured in medium containing 0, 2, 4 or $6 \mu \mathrm{M}$ Anlotinib for $24 \mathrm{~h}$, then (A) TFAP2C mRNA and (B) protein expression levels were measured by RT-qPCR and western blotting, respectively. Data were obtained from three independent repeated experiments. ${ }^{*} \mathrm{P}<0.05$ and ${ }^{* * * *} \mathrm{P}<0.001$ vs. $0 \mu \mathrm{M}$ Anlotinib. MCF-7 cells were transfected with Ov-TFAP2C or Ov-NC, then (C) TFAP2C mRNA and (D) protein expression levels were measured by RT-qPCR and western blotting, respectively. All experiments were independently performed for three times. ${ }^{* * *} \mathrm{P}<0.001$ vs. Ov-NC. (E-H) MCF-7 cells transfected with Ov-TFAP2C or Ov-NC were exposed to $4 \mu \mathrm{M}$ Anlotinib for $24 \mathrm{~h}$, then (E) cell viability was tested using Cell Counting Kit-8 assays, (F and G) the expression of PCNA and Ki-67 was measured by western blotting and $(\mathrm{H})$ cell proliferation was evaluated using colony formation assays. Data were obtained from three independent experiments. ${ }^{* * *} \mathrm{P}<0.001$ vs. control; ${ }^{\# \#} \mathrm{P}<0.01$ and ${ }^{\# \# /} \mathrm{P}<0.001$ vs. Anlotinib + Ov-NC. RT-qPCR, reverse transcription-quantitative PCR; TFAP2C, transcription factor AP-2 $\gamma$; Ov, overexpression; $\mathrm{NC}$, negative control; PCNA, proliferating cell nuclear antigen. 


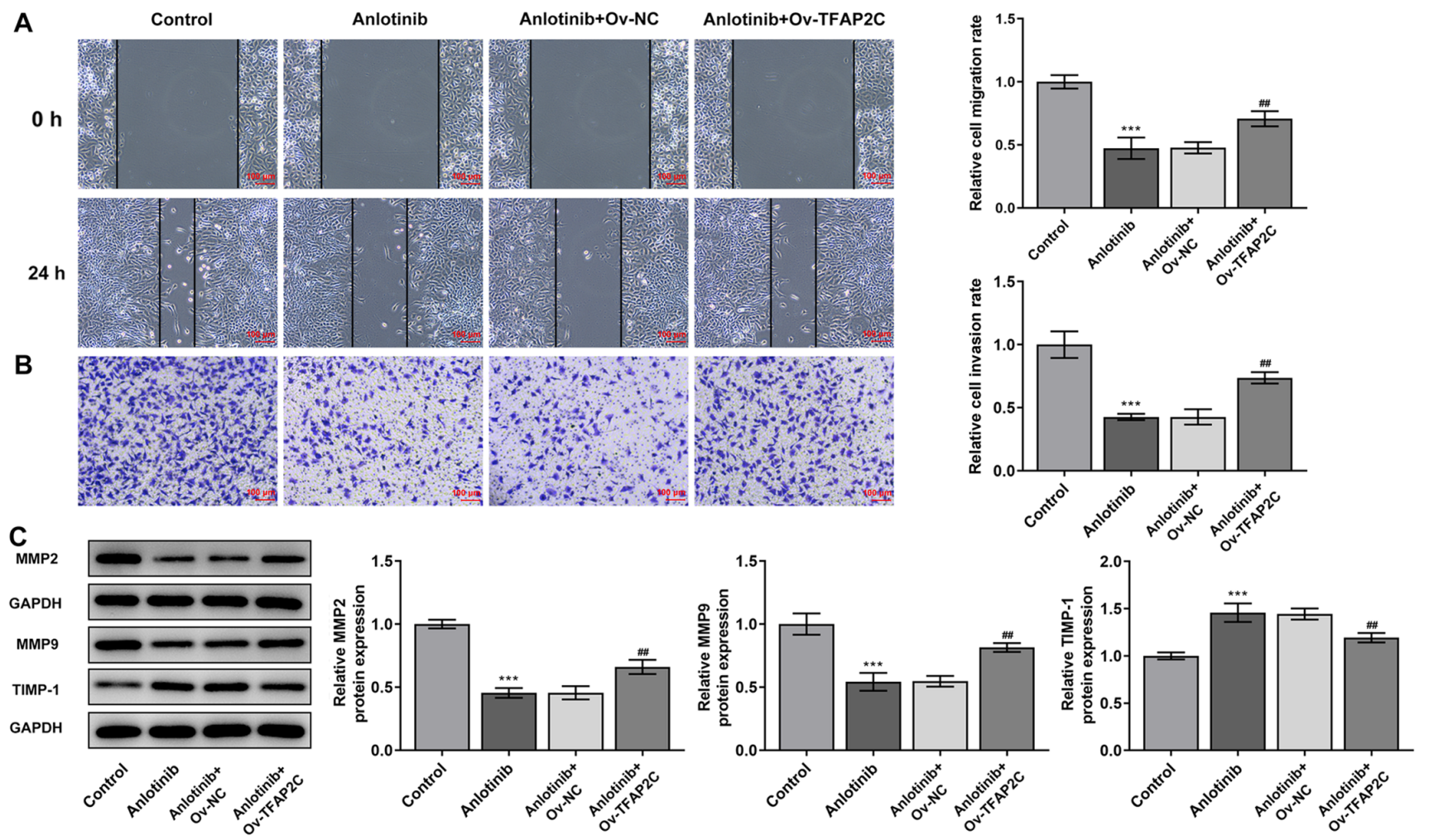

Figure 4. TFAP2C overexpression blocks the effect of Anlotinib on MCF-7 cell migration. MCF-7 cells transfected with Ov-TFAP2C or Ov-NC were exposed to $4 \mu \mathrm{M}$ Anlotinib for $24 \mathrm{~h}$. (A) Cell migration was determined using wound healing and Transwell assays. (B) which were quantified. Scale bars, $100 \mu \mathrm{m}$. (C) MMP2, MMP9 and TIMP-1 protein expression was detected by western blotting. Data were obtained from three independent experiments. ${ }^{* * * *} \mathrm{P}<0.001$ vs. control; ${ }^{\# \#} \mathrm{P}<0.01$ vs. Anlotinib + Ov-NC. TFAP2C, transcription factor AP-2 $\gamma$; Ov, overexpression; NC, negative control; PCNA, proliferating cell nuclear antigen; TIMP-1, tissue inhibitor of metalloproteinase 1.

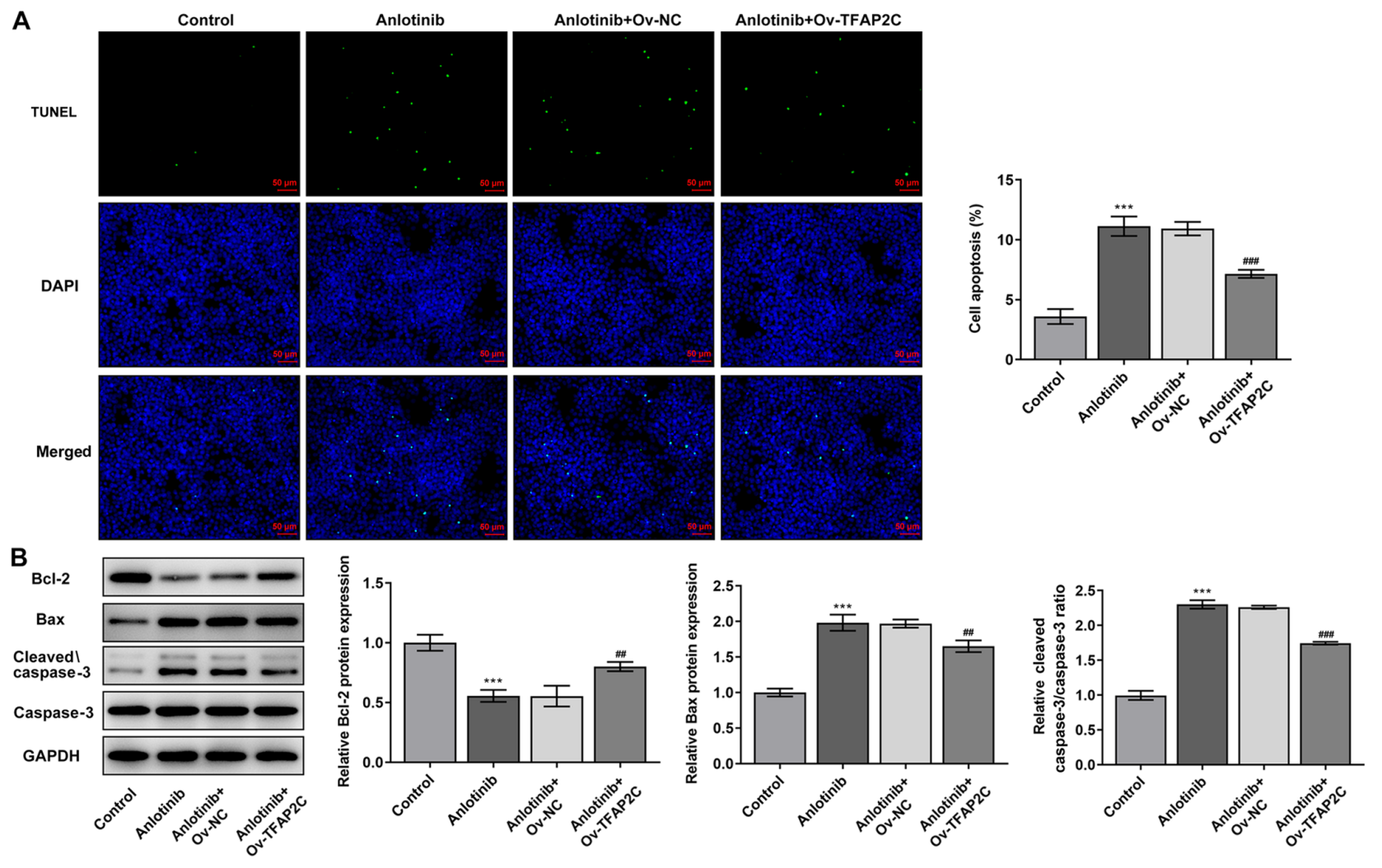

Figure 5. TFAP2C overexpression restores the effect of Anlotinib on MCF-7 cells apoptosis. MCF-7 cells that transfected with Ov-TFAP2C or Ov-NC were exposed to $4 \mu \mathrm{M}$ Anlotinib for $24 \mathrm{~h}$. (A) Apoptosis was observed using TUNEL staining. Scale bars, $50 \mu \mathrm{m}$. (B) Bcl-2, Bax, cleaved caspase-3 and caspase-3 protein expression was measured by western blotting. Data were obtained from three independent experiments. ${ }^{* * *} \mathrm{P}<0.001$ vs. Control; ${ }^{\# \#} \mathrm{P}<0.01$ and ${ }^{\# \# \#} \mathrm{P}<0.001$ vs. Anlotinib + Ov-NC. TFAP2C, transcription factor AP- $2 \gamma$; Ov, overexpression; NC, negative control. 


\section{Acknowledgements}

Not applicable.

\section{Funding}

No funding was received.

\section{Availability of data and materials}

The datasets used and/or analyzed during the current study are available from the corresponding author on reasonable request.

\section{Authors' contributions}

FF and QY designed the experimental study and analyzed the experiment data. FF performed the experiments. FF and QY confirmed the authenticity of all the raw data. All authors have read and approved the final manuscript for submission.

\section{Ethics approval and consent to participate}

Not applicable.

\section{Patient consent for publication}

Not applicable.

\section{Competing interests}

The authors declare that they have no competing interests.

\section{References}

1. DeSantis C, Siegel R, Bandi P and Jemal A: Breast cancer statistics, 2011. CA Cancer J Clin 61: 409-418, 2011.

2. Tajima T, Tokuda T and Kubota M: Treatment of advanced breast cancer: current issues. Cancer and Chemotherapy 25: 1832-1840, 1998 (In Japanese).

3. Maughan KL, Lutterbie MA and Ham PS: Treatment of breast cancer. Am Fam Physician 81: 1339-1346, 2010.

4. Yang J, Yan J and Liu B: Targeting VEGF/VEGFR to modulate antitumor immunity. Front Immunol 9: 978, 2018.

5. Jiao Q, Bi L, Ren Y, Song S, Wang Q and Wang YS: Advances in studies of tyrosine kinase inhibitors and their acquired resistance. Mol Cancer 17: 36, 2018

6. Kerbel RS: Strategies for improving the clinical benefit of antiangiogenic drug based therapies for breast cancer. J Mammary Gland Biol Neoplasia 17: 229-239, 2012.

7. Gao Y, Liu P and Shi R: Anlotinib as a molecular targeted therapy for tumors. Oncol Lett 20: 1001-1014, 2020.

8. Sun Y, Niu W, Du F, Du C, Li S, Wang J, Li L, Wang F, Hao Y, Li C, et al: Safety, pharmacokinetics, and antitumor properties of anlotinib, an oral multi-target tyrosine kinase inhibitor, in patients with advanced refractory solid tumors. J Hematol Oncol 9: 105, 2016.

9. Syed YY: Anlotinib: First global approval. Drugs 78: 1057-1062, 2018.

10. Han B, Li K, Wang Q, Zhang L, Shi J, Wang Z, Cheng Y, He J, Shi Y, Zhao Y, et al: Effect of anlotinib as a third-line or further treatment on overall survival of patients with advanced non-small cell lung cancer: The ALTER 0303 Phase 3 Randomized Clinical Trial. JAMA Oncol 4: 1569-1575, 2018.

11. Li L, Yu J, Jiao S, Wang W, Zhang F and Sun S: Vandetanib (ZD6474) induces antiangiogenesis through mTOR-HIF-1 alphaVEGF signaling axis in breast cancer cells. OncoTargets Ther 11: 8543-8553, 2018.
12. De Andrade JP, Park JM, Gu VW, Woodfield GW, Kulak MV, Lorenzen AW, Wu VT, Van Dorin SE, Spanheimer PM and Weigel RJ: EGFR is regulated by TFAP2C in luminal breast cancer and is a target for vandetanib. Mol Cancer Ther 15: 503-511, 2016.

13. Hu N, Si Y, Yue J, Sun T, Wang X, Jia Z, Gao S, Li Q, Shao Y, Wang J, et al: Anlotinib has good efficacy and low toxicity: A phase II study of anlotinib in pre-treated HER-2 negative metastatic breast cancer. Cancer Biol Med 18: 849-859, 2021.

14. Zhang Y, Wu D, Zhao B, Tian XL, Yao TC, Li F, Liu WF and Shi AP: Application of neoadjuvant chemotherapy combined with anlotinib in occult breast cancer: A case report and review of literature. World J Clin Cases 9: 919-926, 2021.

15. Bogachek MV, Chen Y, Kulak MV, Woodfield GW, Cyr AR, Park JM, Spanheimer PM, Li Y, Li T and Weigel RJ: Sumoylation pathway is required to maintain the basal breast cancer subtype. Cancer Cell 25: 748-761, 2014.

16. Sotiriou C, Wirapati P,Loi S, Harris A, Fox S, Smeds J, Nordgren H, Farmer P, Praz V, Haibe-Kains B, et al: Gene expression profiling in breast cancer: Understanding the molecular basis of histologic grade to improve prognosis. J Natl Cancer Inst 98: 262-272, 2006.

17. Sørlie T, Perou CM, Tibshirani R, Aas T, Geisler S, Johnsen H, Hastie T, Eisen MB, van de Rijn M, Jeffrey SS, et al: Gene expression patterns of breast carcinomas distinguish tumor subclasses with clinical implications. Proc Natl Acad Sci USA 98: 10869-10874, 2001

18. Gu C, Zou S, He C, Zhou J, Qu R, Wang Q, Qi J, Zhou M, Yan S and Ye Z: Long non-coding RNA CCAT1 promotes colorectal cancer cell migration, invasiveness and viability by upregulating VEGF via negative modulation of microRNA-218. Exp Ther Med 19: 2543-2550, 2020.

19. Sui C, Liu D, Hu Y and Zhang L: MicroRNA-708-5p affects proliferation and invasion of osteosarcoma cells by targeting URGCP. Exp Ther Med 17: 2235-2241, 2019.

20. Wang Y, Liu M, Chen S and Wu Q: Plantamajoside represses the growth and metastasis of malignant melanoma. Exp Ther Med 19: 2296-2302, 2020.

21. Livak KJ and Schmittgen TD: Analysis of relative gene expression data using real-time quantitative PCR and the 2(-Delta Delta C(T)) method. Methods 25: 402-408, 2001.

22. Bray F, Ferlay J, Soerjomataram I, Siegel RL, Torre LA and Jemal A: Global cancer statistics 2018: GLOBOCAN estimates of incidence and mortality worldwide for 36 cancers in 185 countries. CA Cancer J Clin 68: 394-424, 2018.

23. Plichta JK, Thomas SM, Vernon R, Fayanju OM, Rosenberger LH, Hyslop T, Hwang ES and Greenup RA: Breast cancer tumor histopathology, stage at presentation, and treatment in the extremes of age. Breast Cancer Res Treat 180: 227-235, 2020.

24. Gouri A, Benarba B, Dekaken A, Aoures H and Benharkat S: Prediction of late recurrence and distant metastasis in early-stage breast cancer: Overview of current and emerging biomarkers. Curr Drug Targets 21: 1008-1025, 2020.

25. Woolston C: Breast cancer. Nature 527: S101, 2015.

26. Franke CM, Gu VW, Grimm BG, Cassady VC, White JR, Weigel RJ and Kulak MV: TFAP2C regulates carbonic anhydrase XII in human breast cancer. Oncogene 39: 1290-1301, 2020.

27. Woodfield GW, Horan AD, Chen Y and Weigel RJ: TFAP2C controls hormone response in breast cancer cells through multiple pathways of estrogen signaling. Cancer Res 67: 8439-8443, 2007.

28. Perkins SM, Bales C, Vladislav T, Althouse S, Miller KD, Sandusky G, Badve S and Nakshatri H: TFAP2C expression in breast cancer: Correlation with overall survival beyond 10 years of initial diagnosis. Breast Cancer Res Treat 152: 519-531, 2015.

29. Wu VT, Kiriazov B, Koch KE, Gu VW, Beck AC, Borcherding N, Li T, Addo P, Wehrspan ZJ, Zhang W, et al: A TFAP2C gene signature is predictive of outcome in HER2-positive breast cancer. Mol Cancer Res 18: 46-56, 2020.

30. Spanheimer PM, Woodfield GW, Cyr AR, Kulak MV, White-Baer LS, Bair TB and Weigel RJ: Expression of the RET proto-oncogene is regulated by TFAP2C in breast cancer independent of the estrogen receptor. Ann Surg Oncol 20: 2204-2212, 2013.

31. Kulak MV, Cyr AR, Woodfield GW, Bogachek M, Spanheimer PM, Li T, Price DH, Domann FE and Weigel RJ: Transcriptional regulation of the GPX1 gene by TFAP2C and aberrant $\mathrm{CpG}$ methylation in human breast cancer. Oncogene 32: 4043-4051, 2013.

This work is licensed under a Creative Commons Attribution-NonCommercial-NoDerivatives 4.0 International (CC BY-NC-ND 4.0) License. 